\title{
THE INTERNAL STABILIZATION BY NOISE OF THE LINEARIZED NAVIER-STOKES EQUATION*
}

\author{
VIOREL BARBU ${ }^{1}$
}

\begin{abstract}
One shows that the linearized Navier-Stokes equation in $\mathcal{O} \subset R^{d}, d \geq 2$, around an unstable equilibrium solution is exponentially stabilizable in probability by an internal noise controller $V(t, \xi)=\sum_{i=1}^{N} V_{i}(t) \psi_{i}(\xi) \dot{\beta}_{i}(t), \xi \in \mathcal{O}$, where $\left\{\beta_{i}\right\}_{i=1}^{N}$ are independent Brownian motions in a probability space and $\left\{\psi_{i}\right\}_{i=1}^{N}$ is a system of functions on $\mathcal{O}$ with support in an arbitrary open subset $\mathcal{O}_{0} \subset \mathcal{O}$. The stochastic control input $\left\{V_{i}\right\}_{i=1}^{N}$ is found in feedback form. One constructs also a tangential boundary noise controller which exponentially stabilizes in probability the equilibrium solution.
\end{abstract}

Mathematics Subject Classification. 35Q30, 60H15, 35B40.

Received January 3rd, 2009. Revised June 17, 2009 and July 23, 2009.

Published online October 30, 2009.

\section{INTRODUCTION}

Consider the Navier-Stokes equation

$$
\begin{array}{ll}
X_{t}-\nu_{0} \Delta X+(X \cdot \nabla) X=f_{e}+\nabla p & \text { in }(0, \infty) \times \mathcal{O} \\
\nabla \cdot X=0,\left.\quad X\right|_{\partial \mathcal{O}}=0 & \text { in } \mathcal{O}, \\
X(0)=x_{0}, &
\end{array}
$$

where $\mathcal{O}$ is an open and bounded subset of $R^{d}, d \geq 2$, with smooth boundary $\partial \mathcal{O}$. Here $f_{e} \in\left(L^{2}(\mathcal{O})\right)^{d}$ is given.

Let $X_{e}$ be an equilibrium solution to (1.1), i.e.,

$$
\begin{aligned}
& -\nu_{0} \Delta X_{e}+\left(X_{e} \cdot \nabla\right) X_{e}=f_{e}+\nabla p_{e} \text { in } \mathcal{O} \\
& \nabla \cdot X_{e}=0 \text { in } \mathcal{O},\left.\quad X_{e}\right|_{\partial \mathcal{O}}=0 .
\end{aligned}
$$

If $X \longrightarrow X-X_{e}$ equation (1.1) reduces to

$$
\begin{aligned}
& X_{t}-\nu_{0} \Delta X+(X \cdot \nabla) X_{e}+\left(X_{e} \cdot \nabla\right) X+(X \cdot \nabla) X=\nabla p \text { in }(0, \infty) \times \mathcal{O}, \\
& \nabla \cdot X=0 \text { in } \mathcal{O},\left.\quad X\right|_{\partial \mathcal{O}}=0, \quad \forall t \geq 0, \\
& X(0)=x \text { in } \mathcal{O},
\end{aligned}
$$

where $x=x_{0}-X_{e}$.

Keywords and phrases. Navier-Stokes equation, feedback controller, stochastic process, Stokes-Oseen operator.

* Supported by CNCSIS project PN II-IDEI-ID_70/2008.

1 Al.I. Cuza University and Octav Mayer Institute of Mathematics of Romanian Academy, Iaşi, Romania. vb41@uaic.ro 
Then, the linearized system around $X_{e}$ associated with (1.1) is the Stokes-Oseen system

$$
\begin{aligned}
& X_{t}-\nu_{0} \Delta X+(X \cdot \nabla) X_{e}+\left(X_{e} \cdot \nabla\right) X=\nabla p \text { in }(0, \infty) \times \mathcal{O} \\
& \nabla \cdot X=0 \text { in } \mathcal{O},\left.\quad X\right|_{\partial \mathcal{O}}=0, \quad t \geq 0 \\
& X(0)=x \text { in } \mathcal{O} .
\end{aligned}
$$

If set $H=\left\{X \in\left(L^{2}(\mathcal{O})\right)^{d} ; \nabla \cdot X=0,\left.X \cdot n\right|_{\partial \mathcal{O}}=0\right\}$, where $\nu$ is the normal to $\partial \mathcal{O}$ and $P:\left(L^{2}(\mathcal{O})\right)^{d} \rightarrow H$ is the Leray projector on $H$, we can rewrite system (1.4) as

$$
\begin{aligned}
& \dot{X}(t)+\mathcal{A} X(t)=0, t \geq 0, \\
& X(0)=x
\end{aligned}
$$

where $\mathcal{A}=\nu_{0} A+A_{0}, A=-P \Delta, D(A)=\left(H_{0}^{1}(\mathcal{O}) \cap H^{2}(\mathcal{O})\right)^{d} \cap H, A_{0}(X)=P\left((X \cdot \nabla) X_{e}+\left(X_{e} \cdot \nabla\right) X\right)$, $D(\mathcal{A})=D(A)$.

Our purpose here is to stabilize (1.5) or, equivalently, the stationary solution $X_{e}$ to (1.1), using a stochastic controller with support in an arbitrary open subset $\mathcal{O}_{0} \subset \mathcal{O}$. To this aim we associate with (1.5) the control stochastic system

$$
\begin{aligned}
& \mathrm{d} X(t)+\mathcal{A} X(t) \mathrm{d} t=\sum_{i=1}^{N} V_{i}(t) \psi_{i} \mathrm{~d} \beta_{i}(t), \\
& X(0)=x,
\end{aligned}
$$

where $\left\{\beta_{i}\right\}_{i=1}^{N}$ is an independent system of real Brownian motions in a probability space $\left\{\Omega, \mathbb{P}, \mathcal{F}, \mathcal{F}_{t}\right\}_{t>0}$. The main results, Theorems 2.1 and 4.1 below, amounts to saying that, in the complexified space $\widetilde{H}$ associated with $H$, under appropriate assumptions on $\mathcal{A}$ (and, implicitly, on $X_{e}$ ), for each $\gamma>0$ there exists $N \in \mathbb{N}$, $\left\{\psi_{i}\right\}_{i=1}^{N} \subset \widetilde{H}$ and an $N$-dimensional adapted process $\left\{V_{i}=V_{i}(t, \omega)\right\}_{i=1}^{N}, \omega \in \Omega$ such that $t \rightarrow \mathrm{e}^{\gamma t} X(t, \omega)$ is convergent to zero in probability for $t \rightarrow \infty$. Moreover, it turns out that the stabilizable controller $\left\{V_{i}\right\}_{i=1}^{i=N}$ can be expressed as a linear feedback controller of the form

$$
V_{i}(t)=\eta\left(X(t), \varphi_{i}^{*}\right)_{\widetilde{H}}, \psi_{i}=P\left(m \phi_{i}\right), i=1, \ldots, N
$$

where $\varphi_{i}^{*}$ are the eigenfunctions of the dual Stokes-Oseen operator $\mathcal{A}^{*}$ corresponding to eigenvalues $\bar{\lambda}_{j}$ with $\operatorname{Re} \lambda_{j} \leq \gamma,\left\{\phi_{i}\right\}_{i=1}^{N}$ is a system of functions related to $\varphi_{i}^{*}$ and $m=\mathcal{X}_{\mathcal{O}_{0}}$ is the characteristic function of $\mathcal{O}_{0}$.

We may view (1.6) as the deterministic system (1.5) perturbed by the white noise controller $\sum_{i=1}^{N} V_{i}(t) \psi_{i} \dot{\beta}_{i}, i . e$.,

$$
\dot{X}+\mathcal{A} X=\sum_{i=1}^{N} V_{i}(t) \psi_{i} \dot{\beta}_{i}
$$

The proof uses some spectral techniques developed in [5,6] (see also $[4,13,14,20,21]$ ) for stabilization of NavierStokes equations. The previous treatment for the stabilization of Navier-Stokes equations is a Riccati based approach which can be described in a few words as follows; one shows first that the unstable finite dimensional part of the Stokes-Oseen equation is stabilizable and one uses this to construct, via the algebraic infinite dimensional Riccati equations associated with the Stokes-Oseen operator, a stabilizable feedback controller. In this context, we note also that in [12] was developed a statistical approach to stabilization of Stokes-Oseen equation in order to treat the unpredictable fluctuations arising in feedback mechanism. This is related to some long-time behaviour results for solutions to Navier-Stokes equations perturbed by random kick-forces (see $[16,22])$. However, the results obtained here are essentially stochastic not only because the stabilizable controller arises as multiplicative term of a Brownian $N$-dimensional motion but mainly because the asymptotic 
nature of stabilization results as well as the stochastic approach have no analogue in deterministic stabilization technique. As a matter of fact, it was known long time ago that one might use the multiplicative noise to stabilize differential systems (see [3]) and more recent results in this direction can be found in [1,2,7-9,19]. (See also [11] for related results.) It must be said however that in the context of Navier-Stokes equations the results obtained here are new. The apparent advantage of the stochastic feedback controller (1.7) compared with deterministic stabilizable controllers constructed by spectral techniques (see $[5,6,13,14,20,21]$ ) is that it avoids the infinite dimensional algebraic Riccati equations which are not numerically tractable by discretization with a larger number of grid points and so are inadequate to treat most fluid dynamic problems with a sufficient degree of resolution. One might suspect that the controller (1.7) is locally stabilizable as well for the Navier-Stokes equation (1.3), and we expect to study this problem in a forthcoming paper.

The plan of the paper is the following. The internal stabilization result, Theorem 2.1, is formulated in Section 2 and proven in Section 3. The boundary stabilization by noise is studied in Section 4.

\section{Notations}

Throughout in the following $\beta_{i}, i=1, \ldots$, are independent real Brownian motions in a probability space $\left\{\Omega, \mathbb{P}, \mathcal{F}, \mathcal{F}_{t}\right\}_{t>0}$ and we shall refer to $[10,17]$ for definition and basic results on stochastic analysis of differential systems and spaces of stochastic processes adapted to filtration $\left\{\mathcal{F}_{t}\right\}_{t>0}$. We shall denote by $\widetilde{H}$ the complexified space $H+\mathrm{i} H$ with scalar product denoted by $\langle\cdot, \cdot\rangle$ and norm by $|\cdot|_{\tilde{H}}$. The scalar product of $H$ is denoted $(\cdot, \cdot)_{H}$ and the norm $|\cdot|_{H} . C_{W}\left([0, T] ; L^{2}(\Omega, \widetilde{H})\right)$ is the space of all adapted square-mean $\widetilde{H}$-valued continuous processes on $[0, T]$.

\section{The MAIN RESUlT}

To begin with, let us briefly recall a few elementary spectral properties of the Stokes-Oseen operator $\mathcal{A}$.

Denote again by $\mathcal{A}$ the extension of $\mathcal{A}$ to the complex space $\widetilde{H}$. The operator $\mathcal{A}$ has a compact resolvent $(\lambda I-\mathcal{A})^{-1}$ and $-\mathcal{A}$ generates a $C_{0}$-analytic semigroup $\mathrm{e}^{-\mathcal{A} t}$ in $\widetilde{H}$. Consequently, $\mathcal{A}$ has a countable number of eigenvalues $\left\{\lambda_{j}\right\}_{j=1}^{\infty}$ with corresponding eigenfunctions $\varphi_{j}$ each with finite algebraic multiplicity $m_{j}$. Of course, certain eigenfunctions $\varphi_{j}$ might be generalized and so, in general, $\mathcal{A}$ is not diagonalizable, i.e., the algebraic multiplicity of $\lambda_{j}$ might not coincide with its geometric multiplicity. Also, each eigenvalue $\lambda_{j}$ will be repeated according to its algebraic multiplicity $m_{j}$.

We shall denote by $N$ the number of eigenvalues $\lambda_{j}$ with $\operatorname{Re} \lambda_{j} \leq \gamma, j=1, \ldots, N$, where $\gamma$ is a fixed positive number.

Denote by $P_{N}$ the projector on the finite dimensional subspace

$$
\mathcal{X}_{u}=\operatorname{lin} \operatorname{span}\left\{\varphi_{j}\right\}_{j=1}^{N} .
$$

We have $\mathcal{X}_{u}=P_{N} \widetilde{H}$ and

$$
P_{N}=-\frac{1}{2 \pi i} \int_{\Gamma}(\lambda I-\mathcal{A})^{-1} \mathrm{~d} \lambda,
$$

where $\Gamma$ is a closed smooth curve in $\mathbb{C}$ which is the boundary of a domain containing in interior the eigenvalues $\left\{\lambda_{j}\right\}_{j=1}^{N}$.

Let $\mathcal{A}_{u}=P_{N} \mathcal{A}, \mathcal{A}_{s}=\left(I-P_{N}\right) \mathcal{A}$. Then $\mathcal{A}_{u}, \mathcal{A}_{s}$ leave invariant the spaces $\mathcal{X}_{u}, \mathcal{X}_{s}=\left(I-P_{N}\right) \widetilde{H}$ and the spectra $\sigma\left(\mathcal{A}_{u}\right), \sigma\left(\mathcal{A}_{s}\right)$ are given by (see $\left.[15]\right)$

$$
\sigma\left(\mathcal{A}_{u}\right)=\left\{\lambda_{j}\right\}_{j=1}^{N}, \quad \sigma\left(\mathcal{A}_{s}\right)=\left\{\lambda_{j}\right\}_{j=N+1}^{\infty} .
$$

Since $\sigma\left(\mathcal{A}_{s}\right) \subset\{\lambda \in \mathbb{C} ; \operatorname{Re} \lambda>\gamma\}$ and $\mathcal{A}_{s}$ generates an analytic $C_{0}$-semigroup on $\widetilde{H}$, we have

$$
\left|\mathrm{e}^{-\mathcal{A}_{s} t} x\right|_{\widetilde{H}} \leq C \mathrm{e}^{-\gamma t}|x|_{\widetilde{H}}, \quad \forall x \in \widetilde{H}, t \geq 0 .
$$


The eigenvalue $\lambda_{j}$ is said to be semi-simple if for it the algebraic and geometrical multiplicity coincides, or, equivalently, $\lambda_{j}$ is a simple pole for $(\lambda I-\mathcal{A})^{-1}$. If all the eigenvalues $\left\{\lambda_{j}\right\}_{j=1}^{N}$ of the matrix $\mathcal{A}_{u}$ are semi-simple, then $\mathcal{A}_{u}$ is diagonalizable.

Herein, we shall assume that the following hypothesis holds.

$\left(\mathrm{A}_{1}\right)$ All the eigenvalues $\lambda_{j}, j=1, \ldots, N$, are semi-simple.

It should be said that hypothesis $\left(A_{1}\right)$ is less restrictive as it might appear to be at first glance. Indeed, it follows by a standard argument involving the Sard-Smale theorem that the property of eigenvalues of the Stokes-Oseen operator to be simple (and, consequently, semi-simple) is generic in the class of coefficients $X_{e}$. So, "almost everywhere" (in the sense of a set of first category), hypothesis $\left(\mathrm{A}_{1}\right)$ holds.

Denote by $\mathcal{A}^{*}$ the adjoint operator and by $P_{N}^{*}$ the adjoint of $P_{N}$. We have

$$
P_{N}^{*}=-\frac{1}{2 \pi i} \int_{\bar{\Gamma}}\left(\lambda I-\mathcal{A}^{*}\right)^{-1} \mathrm{~d} \lambda
$$

The eigenvalues of $\mathcal{A}^{*}$ are precisely the complex conjugates $\bar{\lambda}_{j}$ of eigenvalues $\lambda_{j}$ of $\mathcal{A}$ and they have the same multiplicity. Denote by $\varphi_{j}^{*}$ the eigenfunction of $\mathcal{A}^{*}$ corresponding to the eigenvalue $\lambda_{j}$. We have, therefore,

$$
\mathcal{A} \varphi_{j}=\lambda_{j} \varphi_{j}, \quad \mathcal{A}^{*} \varphi_{j}^{*}=\bar{\lambda}_{j} \varphi_{j}^{*}, \quad j=1, \ldots
$$

Since the eigenvalues $\left\{\lambda_{j}\right\}_{j=1}^{N}$ are semi-simple, it turns out that the system consisting of $\left\{\varphi_{j}\right\}_{j=1}^{N},\left\{\varphi_{j}^{*}\right\}_{j=1}^{N}$ can be chosen to form a bio-orthonormal sequence in $\widetilde{H}$, i.e,

$$
\left\langle\varphi_{j}, \varphi_{i}^{*}\right\rangle=\delta_{i j}, \quad i, j=1, \ldots, N
$$

(see, e.g., [5]). We notice also that the functions $\varphi_{j}$ and $\varphi_{j}^{*}$ have the unique continuation property, i.e.,

$$
\varphi_{j} \not \equiv 0, \quad \varphi_{j}^{*} \not \equiv 0 \text { on } \mathcal{O}_{0} \text { for all } j=1, \ldots, N
$$

(see, e.g., Lem. 3.7 in [5]). We shall assume also that the following condition holds:

$\left(\mathrm{A}_{2}\right)$ The system $\left\{\varphi_{j}^{*}\right\}_{j=1}^{N}$ is linearly independent in $\left(L^{2}\left(\mathcal{O}_{0}\right)\right)^{d}$.

It should be noticed that hypothesis $\left(\mathrm{A}_{2}\right)$ automatically holds if $X_{e}$ is analytic because in this case $\varphi_{j}^{*}$ are analytic too and so $\left(\mathrm{A}_{2}\right)$ is the consequence of linear independence of $\left\{\varphi_{j}^{*}\right\}_{j=1}^{N}$ on $\mathcal{O}_{0}$. Also, in the case where the system $\left\{\varphi_{j}^{*}\right\}_{j=1}^{N}$ contains only one distinct eigenvalue (which might be multiple), hypothesis $\left(\mathrm{A}_{2}\right)$ is implied by the unique continuation property (2.6). It turns out via unique continuation arguments that $\left(\mathrm{A}_{2}\right)$ holds under more general conditions on $X_{e}$ but the presentation of this result is beyond the goals of this work.

Consider the following stochastic perturbation of the linearized system (1.5) considered in the complex space

$$
\begin{aligned}
& \mathrm{d} X+\mathcal{A} X \mathrm{~d} t=\eta \sum_{i=1}^{N}\left\langle X, \varphi_{i}^{*}\right\rangle P\left(m \phi_{i}\right) \mathrm{d} \beta_{i} \\
& X(0)=x
\end{aligned}
$$

where $\eta \in \mathbf{R}$ and $m=\chi_{\mathcal{O}_{0}}$ is the characteristic function of the open subset $\mathcal{O}_{0} \subset \mathcal{O}$. Here $\left\{\phi_{i}\right\}_{i=1}^{N} \subset \widetilde{H}$ is a system of functions to be precised below. We may rewrite (2.7) as

$$
X(t)=\mathrm{e}^{-\mathcal{A} t} x+\eta \sum_{i=1}^{N} \int_{0}^{t}\left\langle X(s), \varphi_{i}^{*}\right\rangle \mathrm{e}^{-\mathcal{A}(t-s)} P\left(m \phi_{i}\right) \mathrm{d} \beta_{i}(s), t \geq 0, \mathbb{P} \text {-a.s. }
$$

which, by the standard existence theory (see $[10])$, has a unique solution $X \in C_{W}\left([0, T] ; L^{2}(\Omega, \widetilde{H})\right), \forall T>0$. 
The closed loop system (2.7) can be equivalently written as (see (1.4))

$$
\begin{aligned}
& \mathrm{d} X(t)-\nu_{0} \Delta X(t) \mathrm{d} t+(X(t) \cdot \nabla) X_{e} \mathrm{~d} t+\left(X_{e} \cdot \nabla\right) X(t) \mathrm{d} t \\
& \quad=\eta m \sum_{i=1}^{N}\left\langle X(t), \varphi_{i}^{*}\right\rangle \phi_{i} \mathrm{~d} \beta_{i}(t)+\nabla p(t) \mathrm{d} t \text { in }(0, \infty) \times \mathcal{O}, \mathbb{P} \text {-a.s. } \\
& \nabla \cdot X(t)=0 \text { in } \mathcal{O},\left.\quad X(t)\right|_{\partial \mathcal{O}}=0, \forall t \geq 0, \mathbb{P} \text {-a.s. } \\
& X(0)=x \text { in } \mathcal{O} .
\end{aligned}
$$

Hence, in the space $\left(L^{2}(\mathcal{O})\right)^{d}$, the feedback controller $\left\{u_{i}=\eta m\left\langle X, \varphi_{i}^{*}\right\rangle \phi_{i}\right\}_{i=1}^{N}$ has the support in $\mathcal{O}_{0}$.

We shall define now $\phi_{j}, j=1, \ldots, N$, as follows:

$$
\phi_{j}(\xi)=\sum_{i=1}^{N} \alpha_{i j} \varphi_{i}^{*}(\xi), \quad \xi \in \mathcal{O}
$$

where $\alpha_{i j}$ are chosen such that

$$
\sum_{i=1}^{N} \alpha_{i j}\left\langle\varphi_{i}^{*}, \varphi_{k}^{*}\right\rangle_{0}=\delta_{j k}, \quad j, k=1, \ldots, N .
$$

(Since, in virtue of hypothesis $\left(\mathrm{A}_{2}\right)$, the matrix $\left\{\left\langle\varphi_{i}^{*}, \varphi_{j}^{*}\right\rangle_{0}\right\}_{i, j=1}^{N}$ is not singular, this is possible.) With this choice, we have

$$
\left\langle\phi_{j}, \varphi_{i}^{*}\right\rangle_{0}=\delta_{i j}, \quad i, j=1, \ldots, N .
$$

Here, we have used the notation $\langle u, v\rangle_{0}=\int_{\mathcal{O}_{0}} u(\xi) \bar{v}(\xi) \mathrm{d} \xi$.

Theorem 2.1 below is the main result.

Theorem 2.1. Under hypotheses $\left(\mathrm{A}_{1}\right),\left(\mathrm{A}_{2}\right)$, the solution $X$ to equation $(2.7)$, where $\left\{\phi_{i}\right\}_{i=1}^{N}$ are given by $(2.10)$, satisfies for $|\eta|$ sufficiently large

$$
\mathbb{P}\left[\lim _{t \rightarrow \infty} \mathrm{e}^{\gamma t}|X(t, x)|_{\widetilde{H}}=0\right]=1, \forall x \in H
$$

Remark 2.2. As mentioned earlier, system (2.9) is written here in the complex space $\widetilde{H}$. If set $X_{1}(t)=\operatorname{Re} X(t)$, $X_{2}(t)=\operatorname{Im} X(t)$, it can be rewritten as a real system in $\left(X_{1}, X_{2}\right)$. In this case, the feedback controller is an implicit stabilizable feedback controller with support in $\mathcal{O}_{0}$ for the real Stokes-Oseen equation (1.4). Of course, if $\lambda_{j}, j=1, \ldots, N$, are real, then we may view $X(t)$ as a real valued function and so, in $(2.12),|X|_{\widetilde{H}}=|X|_{H}$.

\section{Proof of Theorem 2.1}

The idea is to decompose equation (2.7) in a finite dimensional system and an infinite dimensional exponentially stable system. To this end, we set $X_{u}=P_{N} X, X_{s}=\left(I-P_{N}\right) X$ and we shall rewrite equation (2.7) as

$$
\begin{aligned}
& \mathrm{d} X_{u}(t)+\mathcal{A}_{u} X_{u}(t) \mathrm{d} t=\eta P_{N} \sum_{i=1}^{N}\left\langle X_{u}(t), \varphi_{i}^{*}\right\rangle P\left(m \phi_{i}\right) \mathrm{d} \beta_{i}(t), t \geq 0, \mathbb{P} \text {-a.s. } \\
& X_{u}(0)=P_{N} x . \\
& \mathrm{d} X_{s}(t)+\mathcal{A}_{s} X_{s}(t) \mathrm{d} t=\eta\left(I-P_{N}\right) \sum_{i=1}^{N}\left\langle X_{u}(t), \varphi_{i}^{*}\right\rangle P\left(m \phi_{i}\right) \mathrm{d} \beta_{i}(t), t \geq 0, \mathbb{P} \text {-a.s. } \\
& X_{s}(0)=\left(I-P_{N}\right) x .
\end{aligned}
$$


Then, we may represent $X_{u}$ as $X_{u}(t)=\sum_{i=1}^{N} y_{i}(t) \varphi_{i}$ and reduce so equation (3.1) via biorthogonal relations (2.5) and (2.11) to the finite dimensional complex system

$$
\begin{aligned}
& \mathrm{d} y_{j}+\lambda_{j} y_{j} \mathrm{~d} t=\eta y_{j} \mathrm{~d} \beta_{j}, j=1, \ldots, N, t \geq 0, \mathbb{P} \text {-a.s. } \\
& y_{j}(0)=y_{j}^{0},
\end{aligned}
$$

where $y_{j}^{0}=\left\langle P_{N} x, \varphi_{j}^{*}\right\rangle$.

Applying Ito's formula in (3.3) to $\varphi(y)=\mathrm{e}^{\gamma t}|y|^{2}$, we obtain that

$$
\frac{1}{2} \mathrm{~d}\left(\mathrm{e}^{2 \gamma t}\left|y_{j}(t)\right|^{2}\right)+\mathrm{e}^{2 \gamma t}\left(\operatorname{Re} \lambda_{j}-\gamma\right)\left|y_{j}(t)\right|^{2} \mathrm{~d} t=\frac{1}{2} \eta^{2} \mathrm{e}^{2 \gamma t}\left|y_{j}(t)\right|^{2} \mathrm{~d} t+\eta \mathrm{e}^{2 \gamma t}\left|y_{j}(t)\right|^{2} \mathrm{~d} \beta_{j}(t), \quad \text { for } \quad j=1, \ldots, N .
$$

Now, in (3.4) we take $z(t)=\mathrm{e}^{2 \gamma t}\left|y_{j}(t)\right|^{2}$ and get that

$$
\mathrm{d} z+2 \mathrm{e}^{2 \gamma t}\left(\operatorname{Re} \lambda_{j}-\gamma\right)\left|y_{j}\right|^{2} \mathrm{~d} t=\eta^{2} \mathrm{e}^{2 \gamma t}\left|y_{j}\right|^{2} \mathrm{~d} t+2 \eta \mathrm{e}^{2 \gamma t}\left|y_{j}\right|^{2} \mathrm{~d} \beta_{j}, j=1, \ldots, N .
$$

In the latter equation, we shall apply Ito's formula to the function

$$
\phi(r)=(\varepsilon+r)^{\delta}, \text { where } 0<\delta<\frac{1}{2} \text { and } \varepsilon>0 .
$$

We have

$$
\phi^{\prime}(r)=\delta(\varepsilon+r)^{\delta-1}, \phi^{\prime \prime}(r)=\delta(\delta-1)(\varepsilon+r)^{\delta-2}, r>0
$$

and we obtain therefore that

$$
\mathrm{d} \phi(z)=\phi^{\prime}(z) \mathrm{d} z+2 \eta^{2} \mathrm{e}^{4 \gamma t} \phi^{\prime \prime}(z)\left|y_{j}\right|^{4} \mathrm{~d} t .
$$

This yields

$$
\begin{aligned}
\mathrm{d} \phi(z)= & -\delta \mathrm{e}^{2 \gamma t}(\varepsilon+z)^{\delta-1}\left[2\left(\operatorname{Re} \lambda_{j}-\gamma\right)\left|y_{j}(t)\right|^{2} \mathrm{~d} t-\eta^{2}\left|y_{j}\right|^{2} \mathrm{~d} t\right. \\
& \left.-2 \eta\left|y_{j}\right|^{2} \mathrm{~d} \beta_{j}\right]+2 \eta^{2} \delta(\delta-1) \mathrm{e}^{4 \gamma t}(\varepsilon+z)^{\delta-2}\left|y_{j}\right|^{4} \mathrm{~d} t .
\end{aligned}
$$

Now, in the latter equation, if replace $z$ by $\mathrm{e}^{2 \gamma t}\left|y_{j}\right|^{2}$, we obtain that

$$
\begin{aligned}
\mathrm{d}\left(\left(\varepsilon+\mathrm{e}^{2 \gamma t}\left|y_{j}\right|^{2}\right)^{\delta}\right)+2 \delta\left(\varepsilon+\mathrm{e}^{2 \gamma t}\left|y_{j}\right|^{2}\right)^{\delta-1} \mathrm{e}^{2 \gamma t}\left(\operatorname{Re} \lambda_{j}-\gamma\right)\left|y_{j}(t)\right|^{2} \mathrm{~d} t= & 2 \eta^{2}(\delta-1) \delta \mathrm{e}^{4 \gamma t}\left(\varepsilon+\mathrm{e}^{2 \gamma t}\left|y_{j}\right|^{2}\right)^{\delta-2}\left|y_{j}\right|^{2} \mathrm{~d} t \\
& +\eta^{2} \delta \mathrm{e}^{2 \gamma t}\left(\varepsilon+\mathrm{e}^{2 \gamma t}\left|y_{j}\right|^{2}\right)^{\delta-1}\left|y_{j}\right| \mathrm{d} t \\
& +2 \eta \delta \mathrm{e}^{2 \gamma t}\left(\varepsilon+\mathrm{e}^{2 \gamma t}\left|y_{j}\right|^{2}\right)^{\delta-1}\left|y_{j}\right| \mathrm{d} \beta_{j}, j=1, \ldots, N .
\end{aligned}
$$

We set

$$
\begin{aligned}
K_{\varepsilon}^{j}(t)= & 2 \delta \mathrm{e}^{2 \gamma t}\left(\varepsilon+\mathrm{e}^{2 \gamma t}\left|y_{j}\right|^{2}\right)^{\delta-1}\left(\operatorname{Re} \lambda_{j}-\gamma\right)\left|y_{j}(t)\right|^{2} \\
& -\delta \eta^{2} \mathrm{e}^{2 \gamma t}\left(\varepsilon+\mathrm{e}^{2 \gamma t}\left|y_{j}(t)\right|^{2}\right)^{\delta-1}\left|y_{j}(t)\right|^{2} \\
& -2 \delta(\delta-1) \eta^{2} \mathrm{e}^{4 \gamma t}\left(\varepsilon+\mathrm{e}^{2 \gamma t}\left|y_{j}(t)\right|^{2}\right)^{\delta-2}\left|y_{j}(t)\right|^{4}, j=1, \ldots, N .
\end{aligned}
$$

Now, taking into account (3.6), we may rewrite (3.5) as

$$
\left(\varepsilon+\mathrm{e}^{2 \gamma t}\left|y_{j}\right|^{2}\right)^{\delta}+\int_{0}^{t} K_{\varepsilon}^{j}(s) \mathrm{d} s=\left(\varepsilon+\left|y_{j}^{0}\right|^{2}\right)^{\delta}+M_{\varepsilon}^{j}(t), t \geq 0, j=1, \ldots, N, \mathbb{P} \text {-a.s. }
$$


where $M_{\varepsilon}^{j}$ is the following stochastic process

$$
M_{\varepsilon}^{j}(t)=2 \delta \eta \int_{0}^{t} \mathrm{e}^{2 \gamma s}\left|y_{j}(s)\right|^{2}\left(\varepsilon+\mathrm{e}^{2 \gamma s}\left|y_{j}(s)\right|^{2}\right)^{\delta-1} \mathrm{~d} \beta_{j}(s), j=1, \ldots, N .
$$

Taking into account that

$$
\lim _{\varepsilon \rightarrow 0}\left|y_{j}(s)\right|^{2}\left(\varepsilon+\mathrm{e}^{2 \gamma s}\left|y_{j}(s)\right|^{2}\right)^{\delta-1} \mathrm{e}^{2 \gamma s}=\mathrm{e}^{2 \gamma \delta s}\left|y_{j}(s)\right|^{2 \delta}, \mathbb{P} \text {-a.s. }
$$

uniformly on $[0, T]$, we may pass to limit into the stochastic equation (3.7) to get that

$$
\mathrm{e}^{2 \gamma \delta t}\left|y_{j}(t)\right|^{2 \delta}+\int_{0}^{t} K_{j}(s) \mathrm{d} s=\left|y_{j}^{0}\right|^{2 \delta}+M_{j}(t), \mathbb{P} \text {-a.s., } t>0,
$$

where

$$
\begin{aligned}
& K_{j}(t)=\lim _{\varepsilon \rightarrow 0} K_{\varepsilon}^{j}(t)=2 \delta\left(\operatorname{Re} \lambda_{j}-\gamma\right) \mathrm{e}^{2 \gamma \delta t}\left|y_{j}(t)\right|^{2 \delta}+2 \delta(1-2 \delta) \eta^{2} \mathrm{e}^{2 \gamma \delta t}\left|y_{j}(t)\right|^{2 \delta}, \\
& M_{j}(t)=2 \delta \eta \int_{0}^{t} \mathrm{e}^{2 \gamma \delta s}\left|y_{j}(s)\right|^{2 \delta} \mathrm{d} \beta_{j}(s), \mathbb{P} \text {-a.s. }
\end{aligned}
$$

If in (3.8) we take the expectation $E$, we obtain that

$$
\mathrm{e}^{2 \gamma \delta t} E\left|y_{j}(t)\right|^{2 \delta}+E \int_{0}^{t} K_{j}(s) \mathrm{d} s=\left|y_{j}^{0}\right|^{2 \delta}, \quad \forall t \geq 0 .
$$

This yields

$$
2 \delta\left(\eta^{2}(1-2 \delta)+\operatorname{Re} \lambda_{j}-\gamma\right) E \int_{0}^{t} \mathrm{e}^{2 \gamma \delta s}\left|y_{j}(s)\right|^{2 \delta} \mathrm{d} s \leq\left|y_{j}^{0}\right|^{2 \delta}, j=1, \ldots, N,
$$

and, since $0<\delta<\frac{1}{2}$, for all $j=1, \ldots, N$, we get therefore, for $\eta$ sufficiently large,

$$
E \int_{0}^{t} \mathrm{e}^{2 \gamma \delta s}\left|y_{j}(s)\right|^{2 \delta} \mathrm{d} s \leq C, \quad \forall t \geq 0, j=1, \ldots, N
$$

This yields

and, in particular, it follows that

$$
E \int_{0}^{\infty} \mathrm{e}^{2 \gamma \delta s}\left|y_{j}(s)\right|^{2 \delta} \mathrm{d} s<\infty, \quad \forall j=1, \ldots, N,
$$

$$
\int_{0}^{\infty} \mathrm{e}^{2 \gamma \delta s}\left|y_{j}(s)\right|^{2 \delta} \mathrm{d} s<\infty, \mathbb{P} \text {-a.s., } j=1, \ldots, N .
$$

It should be said however that the latter does not imply automatically that $\mathrm{e}^{2 \gamma \delta t}\left|y_{j}(t)\right|^{2 \delta}$ is $\mathbb{P}$-a.s. convergent to zero as $t \rightarrow \infty$ and for this we need to invoke some more sophisticated stochastic argument.

We write

$$
\int_{0}^{t} K_{j}(s) \mathrm{d} s=I_{j}(t)-\left(I_{j}\right)_{1}(t), \quad j=1, \ldots, N, \forall t \geq 0, \mathbb{P} \text {-a.s. }
$$

where

$$
\begin{gathered}
I_{j}(t)=2(1-2 \delta) \delta \eta^{2} \int_{0}^{t} \mathrm{e}^{2 \gamma \delta s}\left|y_{j}(s)\right|^{2 \delta} \mathrm{d} s \\
\left(I_{j}\right)_{1}(t)=2 \delta\left(\gamma-\operatorname{Re} \lambda_{j}\right) \int_{0}^{t} \mathrm{e}^{2 \gamma \delta s}\left|y_{j}(s)\right|^{2 \delta} \mathrm{d} s .
\end{gathered}
$$


Then, we may rewrite $(3.8)$ as

$$
\mathrm{e}^{2 \gamma \delta t}\left|y_{j}(t)\right|^{2 \delta}+I_{j}(t)=\left|y_{j}^{0}\right|^{2 \delta}+\left(I_{j}\right)_{1}(t)+M_{j}(t), t \geq 0, \mathbb{P} \text {-a.s. }
$$

Taking into account that, for each $\varepsilon>0$ and $j=1, \ldots, N, M_{j}(t)$ is a local martingale and $t \rightarrow I_{j}(t)$, $t \rightarrow\left(I_{j}\right)_{1}(t)$ are nondecreasing processes, we see by equation (3.10) that $t \rightarrow \mathrm{e}^{2 \gamma \delta t}\left|y_{j}(t)\right|^{2 \delta}$ is a semi-martingale, as the sum of a local martingale and of an adapted finite variation process (see, e.g., [17]). Then, we may apply to equation (3.10) the following asymptotic result which is a variant of the martingale convergence theorem (see Thm. 7 in [18], p. 139, or Lem. 1 in [2]).

Lemma 3.1. Let $I$ and $I_{1}$ be nondecreasing adapted processes, $Z$ be a nonnegative semi-martingale and $M$ a local martingale such that $E(Z(t))<\infty, \forall t \geq 0, I_{1}(\infty)<\infty, \mathbb{P}$-a.s. and

$$
Z(t)+I(t)=Z(0)+I_{1}(t)+M(t), \forall t \geq 0 .
$$

Then, there is $\lim _{t \rightarrow \infty} Z(t)<\infty, \mathbb{P}$-a.s. and $I(\infty)<\infty, \mathbb{P}$-a.s.

We are going to apply Lemma 3.1 to processes $Z(t)=\mathrm{e}^{2 \gamma \delta t}\left|y_{j}(t)\right|^{2 \delta}, I=I_{j}, I_{1}=\left(I_{j}\right)_{1}, M=M_{j}$ defined above.

In virtue of $(3.9),\left(I_{j}\right)_{1}(\infty)<\infty$. This implies, in virtue of Lemma 3.1, that there exists the limit

$$
\lim _{t \rightarrow \infty}\left(\mathrm{e}^{2 \gamma \delta t}\left|y_{j}(t)\right|^{2 \delta}\right)<\infty, j=1, \ldots, N, \mathbb{P} \text {-a.s. }
$$

Since, by (3.9), $\mathrm{e}^{2 \gamma \delta t}\left|y_{j}\right|^{2 \delta} \in L^{1}(0, \infty)$, P-a.s., the limit in (3.11) is zero. It follows therefore that

$$
\lim _{t \rightarrow \infty} \mathrm{e}^{\gamma t}|y(t)|=0, \mathbb{P} \text {-a.s. }
$$

where $|y|^{2}=\sum_{j=1}^{N}\left|y_{j}\right|^{2}$. We have therefore that

$$
\lim _{t \rightarrow \infty} \mathrm{e}^{2 \gamma t}\left|X_{u}(t)\right|_{\widetilde{H}}^{2}=0, \mathbb{P} \text {-a.s. }
$$

By (3.9) and (3.12), it follows also that

$$
\int_{0}^{\infty} \mathrm{e}^{2 \gamma t}|y(t)|^{2} \mathrm{~d} t<\infty, \mathbb{P} \text {-a.s. }
$$

because, by (3.11), it follows that $\mathrm{e}^{2 \gamma \delta t}|y|^{2 \delta} \in L^{\infty}(0, \infty) \mathbb{P}$-a.s. This yields

$$
\int_{0}^{\infty} \mathrm{e}^{2 \gamma t}\left|X_{u}(t)\right|_{\widetilde{H}}^{2} \mathrm{~d} t<\infty, \mathbb{P} \text {-a.s. }
$$

Next, we come back to the infinite dimensional system (3.2). Since, as seen earlier, the operator $-\mathcal{A}_{s}$ generates a $\gamma$-exponentially stable $C_{0}$-semigroup on $\widetilde{H}$, by the Lyapunov theorem there is $Q \in L(\widetilde{H}, \widetilde{H}), Q=Q * \geq 0$ such that

$$
\operatorname{Re}\left\langle Q x, \mathcal{A}_{s} x-\gamma x\right\rangle=\frac{1}{2}|x|_{\widetilde{H}}^{2}, \forall x \in D\left(\mathcal{A}_{s}\right) .
$$

(We note that though $Q$ is not positively definite in the sense that $\inf \{\langle Q x, x\rangle ;|x|=1\}>0$, we have nevertheless that $\langle Q x, x\rangle>0$ for all $x \neq 0$.) 
Applying Ito's formula in (3.2) to the function $\varphi(x)=\frac{1}{2}\langle Q x, x\rangle$, we obtain that

$$
\begin{aligned}
\frac{1}{2} \mathrm{~d}\left\langle Q X_{s}(t), X_{s}(t)\right\rangle & +\frac{1}{2}\left|X_{s}(t)\right|_{\widetilde{H}}^{2} \mathrm{~d} t+\gamma\left\langle Q X_{s}(t), X_{s}(t)\right\rangle \mathrm{d} t=\frac{1}{2} \eta^{2} \sum_{i=1}^{N}\left(Q Y_{i}(t), Y_{i}(t)\right)_{H} \mathrm{~d} t \\
& +\eta \sum_{i=1}^{N}\left(\left(\operatorname{Re}\left(Q X_{s}(t)\right), \operatorname{Re} Y_{i}(t)\right)_{H}+\left(\operatorname{Im}\left(Q X_{s}(t)\right), \operatorname{Im} Y_{i}(t)\right)_{H}\right) d \beta_{i}(t),
\end{aligned}
$$

where $Y_{i}$ are processes defined by

$$
Y_{i}(t)=\left\langle X_{u}(t), \varphi_{i}^{*}\right\rangle\left(I-P_{N}\right) P\left(m \phi_{i}\right), i=1, \ldots, N
$$

This yields

$$
\begin{aligned}
\mathrm{e}^{2 \gamma t}\left\langle Q X_{s}(t), X_{s}(t)\right\rangle+\int_{0}^{t} \mathrm{e}^{2 \gamma s}\left|X_{s}(s)\right|_{\widetilde{H}}^{2} \mathrm{~d} s= & \left\langle Q\left(I-P_{N}\right) x,\left(I-P_{N}\right) x\right\rangle+\eta^{2} \sum_{i=1}^{N} \int_{0}^{t} e^{2 \gamma s}\left\langle Q Y_{i}(s), Y_{i}(s)\right\rangle \mathrm{d} s \\
& +2 \eta \sum_{i=1}^{N} \int_{0}^{t} \mathrm{e}^{2 \gamma s}\left(\left(\operatorname{Re}\left(Q X_{s}(s)\right), \operatorname{Re} Y_{i}(s)\right)_{H}\right. \\
& \left.+\left(\operatorname{Im}\left(Q X_{s}(s)\right), \operatorname{Im} Y_{i}(s)\right)_{H}\right) \mathrm{d} \beta_{i}(s), t \geq 0, \mathbb{P} \text {-a.s. }
\end{aligned}
$$

We shall once again apply Lemma 3.1 to processes $Z, I, M$ defined below

$$
\begin{aligned}
& Z(t)=\mathrm{e}^{2 \gamma t}\left\langle Q X_{s}(t), X_{s}(t)\right\rangle, \\
& I(t)=\int_{0}^{t} \mathrm{e}^{2 \gamma s}\left|X_{s}(s)\right|_{\widetilde{H}}^{2} \mathrm{~d} s, I_{1}(t)=\eta^{2} \sum_{i=1}^{N} \int_{0}^{t} \mathrm{e}^{2 \gamma s}\left\langle Q Y_{i}, Y_{i}\right\rangle \mathrm{d} s, \\
& M(t)=2 \eta \sum_{i=1}^{N} \int_{0}^{t} \mathrm{e}^{2 \gamma s}\left(\left(\operatorname{Re}\left(Q X_{s}(s)\right), \operatorname{Re} Y_{i}(s)\right)_{H}+\left(\operatorname{Im}\left(Q X_{s}(s)\right), \operatorname{Im} Y_{i}(s)\right)_{H}\right) \mathrm{d} \beta_{j}(s), \mathbb{P} \text {-a.s., } t \geq 0 .
\end{aligned}
$$

Since, by the first step of the proof (see (3.14)), $I_{1}(\infty)<\infty$, we conclude therefore that

$$
\lim _{t \rightarrow \infty} \mathrm{e}^{2 \gamma t}\left\langle Q X_{s}(t), X_{s}(t)\right\rangle=0, \mathbb{P} \text {-a.s. }
$$

and, since $Q$ is positive definite in the sense that $\langle Q x, x\rangle=(Q x, x)_{H}>0$ for all $x \in \widetilde{H}$, we have that

$$
\lim _{t \rightarrow \infty} \mathrm{e}^{\gamma t}\left|X_{s}(t)\right|_{\widetilde{H}}=0, \mathbb{P} \text {-a.s. }
$$

Recalling that $X=X_{u}+X_{s}$ and again invoking (3.13), the latter implies (2.12), thereby completing the proof of Theorem 2.1. 


\section{The tangential BOUNDARY STABILIZATION BY NOISE}

We shall keep the notations of Section 3 .

We come back to the Stokes-Oseen system with boundary controller, i.e.,

$$
\begin{array}{ll}
X_{t}-\nu_{0} \Delta X+(X \cdot \nabla) X_{e}+\left(X_{e} \cdot \nabla\right) X=\nabla p & \text { in }(0, \infty) \times \mathcal{O}, \\
\nabla \cdot X=0 & \text { in }(0, \infty) \times \mathcal{O}, \\
X \cdot \nu=0, \quad X=u & \text { in }(0, \infty) \times \partial \mathcal{O}, \\
X(0)=x & \text { in } \mathcal{O} .
\end{array}
$$

Our purpose here is to stabilize the null solutions to (4.1) by a noise boundary controller $u$ of the form

$$
u=\eta \sum_{i=1}^{N} \frac{\partial \widetilde{\phi}_{i}}{\partial \nu}\left\langle X, \varphi_{i}^{*}\right\rangle \dot{\beta}_{i}
$$

where $N$ is, as above, the number of eigenvalues $\lambda_{j}$ of the operator $\mathcal{A}$ with $\operatorname{Re} \lambda_{j} \leq \gamma$ and $\widetilde{\phi}_{i}$ will be defined below. As in the previous case, $\varphi_{j}^{*}$ are the eigenfunctions of $\mathcal{A}^{*}$ corresponding to $\bar{\lambda}_{j}$ (see $(2.4)$ ) and $\left\{\beta_{i}\right\}_{i=1}^{N}$ is an independent system of real Brownian motions in $\left\{\Omega, \mathbb{P}, \mathcal{F}, \mathcal{F}_{t}\right\}$.

Here, we shall assume that hypothesis $\left(\mathrm{A}_{1}\right)$ holds and also that

$\left(\mathrm{A}_{3}\right)$ The system $\left\{\frac{\partial \varphi_{i}^{*}}{\partial \nu}\right\}_{i=1}^{N}$ is linearly independent in $\left(L^{2}(\partial \mathcal{O})\right)^{d}$.

One might suspect that this property is generic in the class of equilibrium solutions $X_{e}$ as is the case with the following weaker version of $\left(\mathrm{A}_{3}\right)$ :

"Each $\frac{\partial \varphi_{j}^{*}}{\partial \nu}$ is not identically zero on $\partial \mathcal{O} . "$

We set

$$
\mathcal{L} y=-\nu_{0} \Delta y+\left(X_{e} \cdot \nabla\right) y+(y \cdot \nabla) X_{e} \text { in } \mathcal{O}
$$

The Stokes-Oseen system

$$
\begin{array}{ll}
X_{t}+\mathcal{L} X=\nabla p & \text { in }(0, \infty) \times \mathcal{O} \\
\nabla \cdot X=0 & \text { in }(0, \infty) \times \mathcal{O} \\
X \cdot \nu=0, \quad X=u & \text { in }(0, \infty) \times \partial \mathcal{O} \\
X(0)=x & \text { in } \mathcal{O}
\end{array}
$$

can be equivalently written as (see, e.g., [6])

$$
\begin{aligned}
& \frac{\mathrm{d}}{\mathrm{d} t} X(t)+\widetilde{A} X(t)=\widetilde{A}_{\alpha} D u(t), \quad t \geq 0, \\
& X(0)=x,
\end{aligned}
$$

where $y=D u$ is the solution to the equation

$$
\begin{aligned}
& \alpha y+\mathcal{L} y=\nabla p \text { in } \mathcal{O} \\
& \nabla \cdot y=0 \text { in } \mathcal{O}, \quad y=u, y \cdot \nu=0 \text { on } \partial \mathcal{O}
\end{aligned}
$$

and $\alpha>0$ is fixed and sufficiently large. $\left(D:\left(L^{2}(\partial \mathcal{O})\right)^{d} \rightarrow H\right.$ is the Dirichlet map associated with the operator $\mathcal{L}+\alpha I$.)

Indeed, subtracting the latter from (4.4), we obtain

$$
\begin{aligned}
& X_{t}+(\alpha+\mathcal{L})(X-D u)-\alpha X=\nabla p \text { in }(0, \infty) \times \mathcal{O} \\
& \nabla \cdot X=0, \quad X(0)=x \\
& X-D u=0 \text { on }(0, \infty) \times \partial \mathcal{O}
\end{aligned}
$$


and this can be expressed in the form (4.5), where $\widetilde{\mathcal{A}}: \widetilde{H} \rightarrow(D(A))^{\prime}$ is the extension by transposition of $\mathcal{A}=P \mathcal{L}$ to all of $\widetilde{H}$ and with values in $(D(A))^{\prime}$, defined by

$$
\widetilde{\mathcal{A}} y(\varphi)=\int_{\mathcal{O}} y \overline{\mathcal{A}}^{*} \varphi \mathrm{d} \xi=\left\langle y, \mathcal{A}^{*} \varphi\right\rangle, \quad \forall \varphi \in D\left(\mathcal{A}^{*}\right), y \in \widetilde{H},
$$

and $\widetilde{\mathcal{A}}_{\alpha}=\alpha I+\widetilde{\mathcal{A}}$. Here, $(D(A))^{\prime}=\left(D\left(\mathcal{A}^{*}\right)\right)^{\prime}$ is the dual of the space $D(A)$ endowed with the graph norm in pairing induced by $\widetilde{H}$ as pivot space; we have $D(A) \subset \widetilde{H} \subset(D(A))^{\prime}$ algebraically and topologically. It should be noticed that in this formulation, which is standard in boundary control theory, the right hand side of (4.5) is an element of $(D(A))^{\prime}=\left(D\left(\mathcal{A}^{*}\right)\right)^{\prime}$, i.e., roughly speaking is a "pure" distribution on $\mathcal{O}$, which incorporates the boundary control $u$. We note also that (see [6]) the dual $D^{*} \mathcal{A}_{\alpha}^{*}$ of $\mathcal{A}_{\alpha} D$ is given by

$$
D^{*} \mathcal{A}_{\alpha}^{*} \varphi=-\nu_{0} \frac{\partial \varphi}{\partial \nu}, \quad \forall \varphi \in D(A) .
$$

Our aim here is to insert into the controlled system (4.5) a stochastic boundary controller of the form (4.2). Namely, we shall consider the stochastic differential equation

$$
\begin{aligned}
& \mathrm{d} X(t)+\widetilde{\mathcal{A}} X(t) \mathrm{d} t=\eta \sum_{i=1}^{N} \widetilde{\mathcal{A}}_{\alpha} D\left(\frac{\partial \widetilde{\phi}_{i}}{\partial \nu}\right)\left\langle X(t), \varphi_{i}^{*}\right\rangle \mathrm{d} \beta_{i}(t), t \geq 0, \\
& X(0)=x
\end{aligned}
$$

Here, $\left\{\widetilde{\phi}_{i}\right\}_{i=1}^{N}$ is given by $(2.10)$, where $\alpha_{i j}$ are chosen such that

$$
\sum_{i=1}^{N} \alpha_{i j}\left\langle\frac{\partial \varphi_{i}^{*}}{\partial \nu}, \frac{\partial \varphi_{k}^{*}}{\partial \nu}\right\rangle_{1}=\delta_{j k}, \quad j, k=1, \ldots, N
$$

Here, $|\cdot|_{1}=|\cdot|_{\left(L^{2}(\partial \mathcal{O})\right)^{d}}$ and $\langle u, v\rangle_{1}=\int_{\partial \mathcal{O}} \frac{\partial u}{\partial \nu} \frac{\partial \bar{v}}{\partial \nu} \mathrm{d} \xi$. By assumption $\left(\mathrm{A}_{3}\right)$, it is clear that the system $\left\{\widetilde{\phi}_{i}\right\}_{i=1}^{N}$ is well defined and

$$
\left\langle\varphi_{i}^{*}, \widetilde{\phi}_{j}\right\rangle_{1}=\delta_{i j}, \quad i, j=1, \ldots, N
$$

We can, equivalently, write (4.8) as

$$
X(t)=\mathrm{e}^{-\widetilde{\mathcal{A}} t} x+\eta \int_{0}^{t} \sum_{i=1}^{N} \mathrm{e}^{-\widetilde{\mathcal{A}}(t-s)}\left(\widetilde{\mathcal{A}}_{\alpha} D\left(\frac{\partial \widetilde{\phi}_{i}}{\partial \nu}\right)\right)\left\langle X(s), \varphi_{i}^{*}\right\rangle \mathrm{d} \beta_{i}(s) .
$$

Equation (4.10) has a unique solution $X=X(t)$, which is an $\widetilde{H}$-valued continuous process which can be viewed as solution to problem (see [10], p. 244)

$$
\begin{aligned}
& X_{t}-\nu_{0} \Delta X+(X \cdot \nabla) X_{e}+\left(X_{e} \cdot \nabla\right) X=\nabla p \text { in }(0, \infty) \times \mathcal{O} \\
& \nabla \cdot X=0 \text { in }(0, \infty) \times \mathcal{O} \\
& X(0, \xi)=x(\xi) \text { in } \mathcal{O} \\
& X=\sum_{i=1}^{N} \frac{\partial \widetilde{\phi}_{i}}{\partial \nu}\left\langle X, \varphi_{i}^{*}\right\rangle \dot{\beta}_{i} \text { on }(0, \infty) \times \partial \mathcal{O} .
\end{aligned}
$$

In other words, the boundary controller $u=\left.X\right|_{\partial \mathcal{O}}$ is a white noise on $\partial \mathcal{O}$. Moreover, since $\left(\frac{\partial \widetilde{\phi}_{i}}{\partial \nu} \cdot \nu\right) \cdot \nu=0$ on $\partial \mathcal{O}$ (see, e.g., Lem. 3.3 .1 in [6]) this stochastic controller is tangential, i.e., $X \cdot \nu=0$ on $(0, \infty) \times \partial \mathcal{O}$. 
Theorem 4.1. Assume that hypothesis $\left(\mathrm{A}_{1}\right),\left(\mathrm{A}_{3}\right)$ are satisfied. Then, for $|\eta|$ large enough we have for the solution $X$ to (4.8) (equivalently, (4.10))

$$
\mathbb{P}\left[\lim _{t \rightarrow \infty} \mathrm{e}^{\gamma t}\|X(t)\|_{(D(A))^{\prime}}\right]=0 .
$$

In particular, we have

$$
\lim _{t \rightarrow \infty} \mathrm{e}^{\gamma t}\langle X(t), \varphi\rangle=0, \mathbb{P} \text {-a.s., } \forall \varphi \in D(A) .
$$

Proof. We shall argue as in the proof of Theorem 2.1. Namely, as in the previous case, we shall decompose system (4.8) in two parts,

$$
\begin{aligned}
& \mathrm{d} X_{u}+\mathcal{A}_{u} X_{u} \mathrm{~d} t=\eta \widetilde{P}_{N} \sum_{i=1}^{N} \widetilde{\mathcal{A}}_{\alpha}\left(\frac{\partial \widetilde{\phi}_{i}}{\partial \nu}\right)\left\langle X, \varphi_{i}^{*}\right\rangle \mathrm{d} \beta_{i}, \mathbb{P} \text {-a.s. } \\
& X_{u}(0)=P_{N} x \\
& \mathrm{~d} X_{s}+\mathcal{A}_{s} X_{s} \mathrm{~d} t=\eta\left(I-\widetilde{P}_{N}\right) \sum_{i=1}^{N} \widetilde{\mathcal{A}}_{\alpha}\left(\frac{\partial \widetilde{\phi}_{i}}{\partial \nu}\right)\left\langle X, \varphi_{i}^{*}\right\rangle d \beta_{i}, \mathbb{P} \text {-a.s. } \\
& X_{s}(0)=\left(I-P_{N}\right) x .
\end{aligned}
$$

Here $\widetilde{P}_{N}:(D(A))^{\prime} \rightarrow \mathcal{X}_{u}=\operatorname{lin} \operatorname{span}\left\{\varphi_{j}\right\}_{i=1}^{N}$ is the projector defined as in $(2.1)$ and $\mathcal{A}_{u}=\left.\widetilde{P}_{N} \mathcal{A}\right|_{\mathcal{X}_{u}}$, $\mathcal{A}_{s}=\left.\left(I-\widetilde{P}_{N}\right) \mathcal{A}\right|_{\mathcal{X}_{s}}$. The operator $\widetilde{\mathcal{A}}_{s}$ is the extension of $\mathcal{A}_{s}$ to all of $\widetilde{H}$, i.e., $\widetilde{\mathcal{A}}_{s}: \widetilde{H} \rightarrow(D(A))^{\prime}$ is defined by (4.6).

We represent the solution $X_{u}$ to (4.14) as $X_{u}=\sum_{j=1}^{N} y_{j} \varphi_{j}$ and taking into account (2.5), (4.9), we obtain for $\left\{y_{j}\right\}_{j=1}^{N}$ the finite dimensional stochastic system

$$
\begin{aligned}
& \mathrm{d} y_{j}+\lambda_{j} y_{j} \mathrm{~d} t=\eta y_{j} \mathrm{~d} \beta_{j}, \quad j=1, \ldots, N, \\
& y_{j}(0)=y_{j}^{0} .
\end{aligned}
$$

System (4.16) will be treated in the same way as system (3.3). In fact, we get by exactly the same argument as in the proof of Theorem 2.1, that (see (3.12), (3.14))

$$
\begin{aligned}
\lim _{t \rightarrow \infty} \mathrm{e}^{\gamma t}|y(t)| & =0, \quad \mathbb{P} \text {-a.s. } \\
\int_{0}^{\infty} \mathrm{e}^{2 \gamma t}|y(t)|^{2} \mathrm{~d} t<\infty, & \mathbb{P} \text {-a.s. }
\end{aligned}
$$

where $|y|^{2}=\sum_{j=1}^{N} y_{j}^{2}, y=X_{u}$.

Now, coming back to system (4.15), we shall write it as

$$
\begin{array}{ll}
\mathrm{d} X_{s}+\widetilde{\mathcal{A}}_{s} X_{s} \mathrm{~d} t=\eta \sum_{i=1}^{N} Y_{i}(t) \mathrm{d} \beta_{i}, & t \geq 0, \\
Y_{i}(t)=\left(I-\widetilde{P}_{N}\right) \widetilde{\mathcal{A}}_{s} D\left(\frac{\partial \widetilde{\phi}_{i}}{\partial \nu}\right)\left\langle X_{u}(t), \varphi_{i}^{*}\right\rangle, & i=1, \ldots, N .
\end{array}
$$


By (2.2), it follows that

$$
\left\|\mathrm{e}^{-\mathcal{A}_{s} t} x\right\|_{(D(A))^{\prime}} \leq C \mathrm{e}^{-\gamma t}\|x\|_{(D(A))^{\prime}}, \quad \forall x \in(D(A))^{\prime}
$$

and so, by the Lyapunov theorem, there is a self-adjoint, continuous and positive operator $Q=L\left((D(A))^{\prime}\right.$, $\left.(D(A))^{\prime}\right)$ such that

$$
\operatorname{Re}\left\langle Q x, \widetilde{\mathcal{A}}_{s} x\right\rangle_{*}=\gamma\langle Q x, x\rangle_{*}+\frac{1}{2}\|x\|_{(D(A))^{\prime}}^{2}, \quad \forall x \in(D(A))^{\prime}
$$

where $\langle\cdot, \cdot\rangle_{*}$ is the natural scalar product in $(D(A))^{\prime}$.

Applying Ito's formula in (4.19), we obtain that

$$
\begin{aligned}
\frac{1}{2} \mathrm{~d}\left\langle Q X_{s}(t), X_{s}(t)\right\rangle_{*}+\frac{1}{2}\left\|X_{s}(t)\right\|_{(D(A))^{\prime}}^{2} \mathrm{~d} t+\gamma\left\langle Q X_{s}(t), X_{s}(t)\right\rangle_{*} \mathrm{~d} t \\
\quad=\frac{1}{2} \eta^{2} \sum_{i=1}^{N}\left(Q Y_{i}(t), Y_{i}(t)\right)_{H} \mathrm{~d} t+\eta \sum_{i=1}^{N}\left(\left\langle\operatorname{Re}\left(Q X_{s}\right), Y_{i}\right\rangle_{*}+\left\langle\operatorname{Im}\left(Q X_{s}\right), \operatorname{Im} Y_{i}\right\rangle_{*}\right) \mathrm{d} \beta_{i} .
\end{aligned}
$$

This yields

$$
\begin{aligned}
\mathrm{e}^{2 \gamma t}\left\langle Q X_{s}(t), X_{s}(t)\right\rangle_{*}+\int_{0}^{t} \mathrm{e}^{2 \gamma s}\left\|X_{s}(s)\right\|_{(D(A))^{\prime}}^{2} \mathrm{~d} s= & \left\langle Q\left(I-P_{N}\right) x,\left(I-P_{N}\right) x\right\rangle_{*}+2 \eta \sum_{i=1}^{N} \int_{0}^{t} \mathrm{e}^{2 \gamma s}\left\langle Q Y_{i}(s), Y_{i}(s)\right\rangle_{*} \mathrm{~d} s \\
& +2 \eta \sum_{i=1}^{N} \int_{0}^{t} \mathrm{e}^{2 \gamma s}\left(\left\langle\operatorname{Re}\left(Q X_{s}\right), \operatorname{Im} Y_{i}\right\rangle_{*}+\left\langle\operatorname{Im}\left(Q X_{s}\right), \operatorname{Im} Y_{i}\right\rangle_{*}\right) \mathrm{d} \beta_{i} .
\end{aligned}
$$

Then, applying Lemma 3.1 exactly as in the proof of Theorem 2.1, we infer that

$$
\mathbb{P}\left[\lim _{t \rightarrow \infty}\left\langle Q X_{s}(t), X_{s}(t)\right\rangle_{*} \mathrm{e}^{2 \gamma t}=0\right]=1
$$

and, since $\langle Q x, x\rangle_{*}=0$ implies $x=0$, we infer that

$$
\mathbb{P}\left[\lim _{t \rightarrow \infty} \mathrm{e}^{\gamma t}\left|X_{s}(t)\right|_{(D(A))^{\prime}}=0\right]=1
$$

as claimed. This completes the proof.

Acknowledgements. The author is indebted to the anonymous referees for pertinent comments and remarks which permitted the improvement of this work.

\section{REFERENCES}

[1] J.A.D. Apleby, X. Mao and A. Rodkina, Stochastic stabilization of functional differential equations. Syst. Control Lett. 54 (2005) 1069-1081.

[2] J.A.D. Apleby, X. Mao and A. Rodkina, Stabilization and destabilization of nonlinear differential equations by noise. IEEE Trans. Automat. Contr. 53 (2008) 683-691.

[3] L. Arnold, H. Craul and V. Wihstutz, Stabilization of linear systems by noise. SIAM J. Contr. Opt. 21 (1983) $451-461$.

[4] V. Barbu, Feedback stabilization of Navier-Stokes equations. ESAIM: COCV 9 (2003) 197-205.

[5] V. Barbu and R. Triggiani, Internal stabilization of Navier-Stokes equations with finite dimensional controllers. Indiana Univ. Math. J. 53 (2004) 1443-1494.

[6] V. Barbu, I. Lasiecka and R. Triggiani, Tangential boundary stabilization of Navier-Stokes equations, Memoires Amer. Math. Soc. AMS, USA (2006)

[7] T. Caraballo, K. Liu and X. Mao, On stabilization of partial differential equations by noise. Nagoya Math. J. 101 (2001) $155-170$.

[8] T. Caraballo, H. Craul, J.A. Langa and J.C. Robinson, Stabilization of linear PDEs by Stratonovich noise. Syst. Control Lett. 53 (2004) 41-50. 
[9] S. Cerrai, Stabilization by noise for a class of stochastic reaction-diffusion equations. Prob. Th. Rel. Fields 133 (2000) 190-214.

[10] G. Da Prato, An Introduction to Infinite Dimensional Analysis. Springer-Verlag, Berlin, Germany (2006).

[11] H. Ding, M. Krstic and R.J. Williams, Stabilization of stochastic nonlinear systems driven by noise of unknown covariance. IEEE Trans. Automat. Contr. 46 (2001) 1237-1253.

[12] J. Duan and A. Fursikov, Feedback stabilization for Oseen Fluid Equations. A stochastic approach. J. Math. Fluids Mech. 7 (2005) 574-610.

[13] A. Fursikov, Real processes of the 3-D Navier-Stokes systems and its feedback stabilization from the boundary, in $A M S$ Translations, Partial Differential Equations, M. Vîshnik Seminar 206, M.S. Agranovic and M.A. Shubin Eds. (2002) 95-123.

[14] A. Fursikov, Stabilization for the 3-D Navier-Stokes systems by feedback boundary control. Discrete Contin. Dyn. Syst. 10 (2004) 289-314.

[15] T. Kato, Perturbation Theory of Linear Operators. Springer-Verlag, New York, Berlin (1966).

[16] S. Kuksin and A. Shirikyan, Ergodicity for the randomly forced $2 D$ Navier-Stokes equations. Math. Phys. Anal. Geom. 4 (2001) 147-195.

[17] T. Kurtz, Lectures on Stochastic Analysis. Lecture Notes Online, Wisconsin (2007), available at http://www. math.wisc.edu/ kurtz/735/main735.pdf.

[18] R. Lipster and A.N. Shiraev, Theory of Martingals. Dordrecht, Kluwer (1989).

[19] X.R. Mao, Stochastic stabilization and destabilization. Syst. Control Lett. 23 (2003) 279-290.

[20] J.P. Raymond, Feedback boundary stabilization of the two dimensional Navier-Stokes equations. SIAM J. Contr. Opt. 45 (2006) 790-828.

[21] J.P. Raymond, Feedback boundary stabilization of the three dimensional incompressible Navier-Stokes equations. J. Math. Pures Appl. 87 (2007) 627-669.

[22] A. Shirikyan, Exponential mixing 2D Navier-Stokes equations perturbed by an unbounded noise. J. Math. Fluids Mech. 6 (2004) 169-193. 